\title{
Reply to Comment on "Noise in the Helical Edge Channel Anisotropically Coupled to a Local Spin" (JETP Letters 108, 664 (2018))
}

\author{
K. E. Nagaev ${ }^{a, *}$, S. V. Remizov ${ }^{a, b}$, and D. S. Shapiro ${ }^{a, b}$ \\ ${ }^{a}$ Kotelnikov Institute of Radioengineering and Electronics, Russian Academy of Sciences, Moscow, 125009 Russia \\ ${ }^{b}$ All-Russia Research Institute of Automatics (VNIIA), Moscow, 127055 Russia \\ *e-mail: knagaev@inbox.ru \\ Received March 19, 2019; revised March 19, 2019; accepted March 21, 2019
}

DOI: $10.1134 / \mathrm{S} 0021364019090030$

The authors of comment [1] claim that our recent results [2] on the noise in the helical edge channel of a $2 \mathrm{D}$ topological insulator coupled to a spin-1/2 impurity are incorrect. Their argument is that the expression for the average backscattering current that follows from our Eq. (7) differs from Eq. (22) in their own paper [3]. They state that it is illegal to assume that the density matrix of the impurity spin is diagonal in the basis of $S_{z}$, which is the cornerstone of our calculations and the calculations in [4].

The authors of the comment reason that the dephasing of the impurity spin arises not only from the term $J_{z} S_{z} s_{z}$ in the Hamiltonian, but also from the term $2 J_{a} S_{x} s_{z}$. However this depends on the relative magnitude of the parameters $J_{z}$ and $J_{a}$. In our paper we clearly state that the dephasing of the impurity spin is due to the term $J_{z} S_{z} s_{z}$, and this implies that $J_{z}$ is large. This does not mean that the exchange matrix is diagonal as stated in [1], but only means that $J_{33}$ in Eq. (1) in the comment is much larger than all the other elements of the matrix. Note that this parameter does not enter into any of the transition rates $\Gamma_{0}^{ \pm}, \Gamma_{a}, \Gamma_{1}$, or $\Gamma_{2}$ and its large value does not impose any restrictions on the relations between these quantities.

The authors of the comment admit that in this approximation, their Eq. (5) crosses over to Eq. (7) in our paper [2] written for $J_{2}=J_{a}=0$. In addition, it is clearly seen that the voltage-proportional current in the limit of $J_{2}=J_{1}=0$ and $e V \gg T$, Eq. (3) of the comment, vanishes in this case. Hence, there is no contradiction between [2] and [3].

To summarize, our results are correct within the limits of applicability of our model, and their critique by the authors of the comment is irrelevant.

\section{REFERENCES}

1. I. S. Burmistrov, P. D. Kurilovich, and V. D. Kurilovich, JETP Lett. 109 (2019, in press).

2. K. E. Nagaev, S. V. Remizov, and D. S. Shapiro, JETP Lett. 108, 664 (2018); arXiv: 1810.05831.

3. P. D. Kurilovich, V. D. Kurilovich, I. S. Burmistrov, and M. Goldstein, JETP Lett. 106, 593 (2017).

4. L. Kimme, B. Rosenow, and A. Brataas, Phys. Rev. B 93, 081301 (2016). 